\title{
Bi/BiOCl Nanosheets Enriched with Oxygen Vacancies to Enhance Photocatalytic $\mathrm{CO}_{2}$ Reduction
}

\author{
Shuqi Wu ${ }^{1}$. Junbu Wang ${ }^{1}$ - Qingchuan $\mathrm{Li}^{1} \cdot$ Zeai Huang ${ }^{1,2} \cdot$ Zhiqiang Rao $^{1,2} \cdot$ Ying Zhou $^{1,2}$
}

Received: 16 October 2020 / Revised: 23 October 2020 / Accepted: 3 December 2020 / Published online: 6 January 2021

(c) The Author(s) 2021

\begin{abstract}
$\mathrm{BiOCl}$ has been used in the photoreduction of $\mathrm{CO}_{2}$, but exhibits limited photocatalytic activity. In this study, Bi was in situ reduced and deposited on the surface of (001)-dominated $\mathrm{BiOCl}$ nanosheets by $\mathrm{NaBH}_{4}$ to form $\mathrm{Bi} / \mathrm{BiOCl}$ nanosheets enriched with oxygen vacancies. The as-prepared $\mathrm{Bi} / \mathrm{BiOCl}$ nanosheets having low thickness (ca. $10 \mathrm{~nm}$ ) showed much higher concentration of oxygen vacancies compared to $\mathrm{Bi} / \mathrm{BiOCl}$ nanoplates having high thickness (ca. $100 \mathrm{~nm}$ ). Subsequently, the photocatalytic activity of the $\mathrm{Bi} / \mathrm{BiOCl}$ nanosheets enriched with oxygen vacancies for $\mathrm{CO}_{2}$ reduction was dramatically enhanced and much higher than that of $\mathrm{BiOCl}$ nanoplates, nanosheets, and $\mathrm{Bi} / \mathrm{BiOCl}$ nanoplates. It showed that the improved photocatalytic activity in the reduction of $\mathrm{CO}_{2}$ can be attributed to the enhanced separation efficiency of photogenerated electron-hole pairs of the oxygen vacancies on $\mathrm{BiOCl}$ nanosheets and $\mathrm{Bi}$ metals. This work demonstrated that the in situ reduction of non-noble metals on the surface of $\mathrm{BiOCl}$ nanosheets that are enriched with oxygen vacancies is favorable for increasing photocatalytic $\mathrm{CO}_{2}$ reduction.
\end{abstract}

Keywords $\mathrm{Bi} \cdot$ Oxygen vacancy $\cdot \mathrm{BiOCl}$ nanosheets $\cdot$ Photocatalysis $\cdot \mathrm{CO}_{2}$ reduction

\section{Introduction}

With the development of modern economy and society, fossil fuels are extensively used in many sectors. The burning of fossil fuels inevitably leads to the emission of huge amounts of carbon dioxide $\left(\mathrm{CO}_{2}\right)$. It is predicted that the global concentration of $\mathrm{CO}_{2}$ will reach $500 \mathrm{ppm}$ by the end of the twenty-first century and global temperature will increase by $1.9^{\circ} \mathrm{C}$ [1]. The increasing temperature will cause global warming, which can cause huge economic losses and threaten human survival [2]. Therefore, reducing

Supplementary Information The online version contains supplementary material available at https://doi.org/10.1007/s1220 9-020-00280-6.

Zeai Huang

zeai.huang@swpu.edu.cn

Ying Zhou

yzhou@swpu.edu.cn

1 School of New Energy and Materials, Southwest Petroleum University, Chengdu 610500, China

2 State Key Laboratory of Oil and Gas Reservoir Geology and Exploitation, Southwest Petroleum University, Chengdu 610500, China
$\mathrm{CO}_{2}$ emissions is an urgent necessity. Among the numerous strategies developed to reduce $\mathrm{CO}_{2}$ emission, capturing and utilizing $\mathrm{CO}_{2}$ to produce other carbon sources is a promising way, which has been demonstrated, to reduce atmospheric $\mathrm{CO}_{2}$ concentration. Specifically, since the 1980 s, photocatalytic conversion of $\mathrm{CO}_{2}$ has been reported as an important strategy for $\mathrm{CO}_{2}$ utilization [3]. Until now, various photocatalysts [4-9] have been employed for the process of $\mathrm{CO}_{2}$ utilization. However, the photocatalytic activities of these photocatalysts are still unsatisfactory.

Bismuth oxychloride (BiOCl), a typical two-dimensional (2D) material with a layered structure, has been used for the photoreduction of $\mathrm{CO}_{2}$ [10-12]. However, the $\mathrm{CO}_{2}$ reduction efficiency of $\mathrm{BiOCl}$ photocatalysts is usually quite low because of the following reasons: limited light absorption capacity, low efficiency of electron-hole carriers, and fast recombination of photogenerated electron-hole pairs. Various strategies, such as controlling exposed active facets [12-14], forming oxygen vacancies [10,11, 15, 16], loading metals as cocatalysts [17-19], and constructing heterojunctions [20-23], are used to improve the photocatalytic efficiency of $\mathrm{BiOCl}$ catalysts. The strategy of loading metals as cocatalysts onto $\mathrm{BiOCl}$ to enhance photocatalytic activity has gained increasing attention because cocatalysts can 
form Schottky barriers as strong electron traps to direct the flow of electrons. Recently, a couple of studies have reported bismuth (Bi) as a cocatalyst loaded on $\mathrm{BiOCl}(\mathrm{Bi} / \mathrm{BiOCl})$ to show enhanced photocatalytic activity [24-26]. The use of $\mathrm{Bi}$ as a cocatalyst is not only due to the low cost, but also because Bi can be easily formed in situ on the surface of $\mathrm{BiOCl}$ via reduction reagents. The lattice matching interfaces between $\mathrm{Bi}$ and $\mathrm{BiOCl}$ can be formed in $\mathrm{Bi} / \mathrm{BiOCl}$ to achieve better charge transfer compared with other metals $[27,28]$. Moreover, the in situ reduction process usually induces oxygen vacancies on the surface of $\mathrm{BiOCl}[24,26]$. Oxygen vacancies are believed to favor $\mathrm{CO}_{2}$ adsorption and activation $[11,29,30]$. Therefore, it is possible to develop $\mathrm{Bi} / \mathrm{BiOCl}$ catalysts that are enriched with oxygen vacancies for photocatalytic $\mathrm{CO}_{2}$ reduction.

Herein, photocatalytic $\mathrm{CO}_{2}$ reduction using $\mathrm{Bi}$ metal deposited on $\mathrm{BiOCl}$ nanosheets that were enriched with oxygen vacancies was studied for the first time. It was found that the concentration of oxygen vacancies on $\mathrm{Bi} /$ $\mathrm{BiOCl}$ nanosheets with a thickness of ca. $10 \mathrm{~nm}$ were much higher than on $\mathrm{Bi} / \mathrm{BiOCl}$ nanoplates with a thickness of ca. $100 \mathrm{~nm}$. Bi/BiOCl nanosheets with enriched oxygen vacancies showed much higher photocatalytic activity during $\mathrm{CO}_{2}$ reduction than $\mathrm{BiOCl}$ nanoplates, nanosheets, and $\mathrm{Bi} / \mathrm{BiOCl}$ nanoplates.

\section{Experimental}

\section{Chemicals}

Polyvinyl pyrrolidone (PVP) was purchased from Sigma Co., Ltd, Shanghai, China. All the other reagents were purchased from Chengdu Kelong Chemical Co., Ltd, Chengdu, China. All chemicals were used without further purification, if not specifically mentioned. Ultrapure water was used throughout the experiment.

\section{Preparation of Photocatalysts}

\section{Preparation of BiOCI Nanosheets}

Thin BiOCl nanosheets were synthesized using a method similar to the method reported in Ref. [14]. In a typical synthesis procedure, $1.0 \mathrm{mmol} \mathrm{Bi}\left(\mathrm{NO}_{3}\right)_{3} \cdot 5 \mathrm{H}_{2} \mathrm{O}$ and $1.0 \mathrm{mmol}$ $\mathrm{NaCl}$ were dissolved in a solution containing $30 \mathrm{~mL}$ ethylene glycol and $5 \mathrm{~mL}$ deionized water with strong stirring. Next, $0.2 \mathrm{~g}$ PVP was added into the above suspension. After $30 \mathrm{~min}$ of stirring, the mixture was transferred into $50-\mathrm{mL}$ Teflon-lined autoclave and then heated at $160{ }^{\circ} \mathrm{C}$ for $8 \mathrm{~h}$. After being naturally cooled down to room temperature under air atmosphere, the resultant precipitate was washed with ethanol and deionized water by centrifugation. Then, the mixture was stirred for $20 \mathrm{~min}$ and frozen at $-80^{\circ} \mathrm{C}$ for $2 \mathrm{~h}$. After $48 \mathrm{~h}$ of freeze-drying, the powders were ground and obtained. Finally, the products were calcined at $400{ }^{\circ} \mathrm{C}$ under air atmosphere for $4 \mathrm{~h}$. The resulting thin $\mathrm{BiOCl}$ nanosheets were defined as BiOCl-NS.

\section{Preparation of BiOCI Nanoplates}

The $\mathrm{BiOCl}$ nanoplates were synthesized using water as solvent and without PVP. The $1.0 \mathrm{mmol} \mathrm{Bi}\left(\mathrm{NO}_{3}\right)_{3} \cdot 5 \mathrm{H}_{2} \mathrm{O}$ and $1.0 \mathrm{mmol} \mathrm{NaCl}$ were dissolved in $35 \mathrm{~mL}$ deionized water with strong stirring. After $30 \mathrm{~min}$ of stirring, the mixture was transferred into a 50-mL Teflon-lined autoclave and then heated at $160{ }^{\circ} \mathrm{C}$ for $8 \mathrm{~h}$. The $\mathrm{BiOCl}$ nanoplate was obtained using the post-processing process for $\mathrm{BiOCl}-\mathrm{NS}$, which was defined as BiOC1-NP.

\section{Preparation of $\mathrm{Bi} / \mathrm{BiOCl}$ Nanocomposite}

The $\mathrm{Bi} / \mathrm{BiOCl}$ nanocomposite was obtained via an in situ reduction process using $\mathrm{NaBH}_{4}$ solution. In detail, $0.2 \mathrm{~g}$ of the as-prepared thin $\mathrm{BiOCl}$ nanosheets was added to $30 \mathrm{~mL}$ $\mathrm{NaBH}_{4}$ solution of $4.0 \mathrm{mmol} / \mathrm{L}$ and reacted for $8 \mathrm{~min}$ under stirring. The resulting products were washed with ethanol and deionized water several times until the impurities were removed. Using the same method, the final $\mathrm{Bi} / \mathrm{BiOCl}$ nanocomposites were obtained by freeze-drying. The $\mathrm{Bi} / \mathrm{BiOCl}$ nanocomposite synthesized with various concentrations of $\mathrm{NaBH}_{4}$ solutions was defined as BiOCl-NS. In addition, Bi/ $\mathrm{BiOCl}-\mathrm{NP}$ meant that $4.0 \mathrm{mmol} / \mathrm{L} \mathrm{NaBH}_{4}$ and $\mathrm{BiOCl}$ nanoplates were used during the reduction process.

\section{Characterizations}

Powder X-ray diffraction (XRD) patterns were measured on a PANalytical $\mathrm{X}$ 'pert at $40 \mathrm{kV}$ and $40 \mathrm{~mA}$ with $\mathrm{Cu} \mathrm{K} \alpha$ radiation, and the $2 \theta$ range was measured from $10^{\circ}$ to $70^{\circ}$ with step width of $0.05^{\circ}$. Scanning electron microscopy (SEM) was conducted using JEOL JSM-7800F. Transmission electron microscopy (TEM) was performed using FEI Tecnai $\mathrm{G}^{2}$ F30. The binding environment of the samples and surface chemical composition were investigated using a Thermo ESCALAB250Xi X-ray photoelectron spectroscopy (XPS). Meanwhile, all the binding energies were referenced to the C $1 s$ level at $284.8 \mathrm{eV}$. UV-Vis diffuse reflectance spectra (DRS) were investigated at room temperature using $\mathrm{BaSO}_{4}$ as the standard background for reflectance. The photoluminescence (PL) spectra were recorded on Nicolet 6700 with an excitation wavelength of $370 \mathrm{~nm}$. The electron spin-resonance spectroscopy (ESR) was recorded on a JESFA200 spectrometer at room temperature. 


\section{Photocatalytic Performance}

Photocatalytic reduction of $\mathrm{CO}_{2}$ was conducted in a closed cylindrical reaction vessel with a volume of $380 \mathrm{~mL} .20 \mathrm{mg}$ samples were evenly spread into the culture dish, which had a diameter of $7.5 \mathrm{~cm}$. Then, the dish was put into the reactor and sealed with a stainless steel cover and a quartz window. Next, the reactor was evacuated by a vacuum pump and then high purity Ar gas was introduced. The above steps were repeated for 10 times to eliminate the air in the reactor and to fill the reactor with high purity $\mathrm{Ar} .400 \mathrm{ppm}$ of $\mathrm{CO}_{2}$ was then passed through a two-hole gas wash bottle with $50 \mathrm{~mL}$ of ultrapure water. The $\mathrm{CO}_{2}$ containing water vapor was continuously introduced into the reactor for around $15 \mathrm{~min}$. After unplugging the interface, the reactor was tightly closed. Finally, the mixture of catalyst and $\mathrm{CO}_{2}$ with water vapor was illuminated using a $300-\mathrm{W}$ Xe lamp as a UV-Vis light. The gas products were detected by a Techcomp GC7900 gas chromatograph (GC). A thermal conductivity detector (TCD) was used to detect $\mathrm{H}_{2}$ gas, and a flame ionization detector (FID) was used to detect $\mathrm{CO}$ and $\mathrm{CH}_{4}$ gases. The selectivity toward products was calculated using the following equations:

CO selectivity $(\%)=100 \times 2 R_{\mathrm{CO}} /\left(2 R_{\mathrm{CO}}+2 R_{\mathrm{H}_{2}}+8 R_{\mathrm{CH}_{4}}\right)$
$\mathrm{CH}_{4}$ selectivity (\%) $=100 \times 8 R_{\mathrm{CH}_{4}} /\left(2 R_{\mathrm{CO}}+2 R_{\mathrm{H}_{2}}+8 R_{\mathrm{CH}_{4}}\right)$

$\mathrm{H}_{2}$ selectivity $(\%)=100 \times 2 R_{\mathrm{H}_{2}} /\left(2 R_{\mathrm{CO}}+2 R_{\mathrm{H}_{2}}+8 R_{\mathrm{CH}_{4}}\right)$

\section{Results and Discussion}

Figure 1a shows the XRD spectra of the as-synthesized $\mathrm{BiOCl}$ and $\mathrm{Bi} / \mathrm{BiOCl}$. Both spectra of $\mathrm{BiOCl}$ prepared without reduction were perfectly assigned to tetragonal phase of BiOCl (JCPDS-ICDD card No.06-0249). The diffraction peaks of Bi element (JCPDS-ICDD card No.44-1246) were observed in the samples prepared after $\mathrm{NaBH}_{4}$ reducing. It is noted that the (001) plane assigned to $\mathrm{BiOCl}$ at $11.98^{\circ}$ showed obvious decrease of relative intensity, indicating that Bi might be formed on $\{001\}$ facets. No obvious peak shifts were found in the $\mathrm{Bi} / \mathrm{BiOCl}$ samples reduced by $\mathrm{NaBH}_{4}$ (Fig. 1b), indicating that $\mathrm{Bi}$ elements were not doped into $\mathrm{BiOCl}$.

The micromorphologies of the as-prepared BiOCl-NS and Bi/BiOCl-NS were characterized using TEM (Fig. 2). The pristine BiOCl-NS using EG as a solvent showed nanosheetlike morphology with the length and width are ca. $100 \mathrm{~nm}$ (Fig. 2a, b). The measured thicknesses of these nanosheets were around 8-28 nm (Fig. S1). However, BiOCl-NP prepared using $\mathrm{H}_{2} \mathrm{O}$ as a solvent exhibited a thickness of a
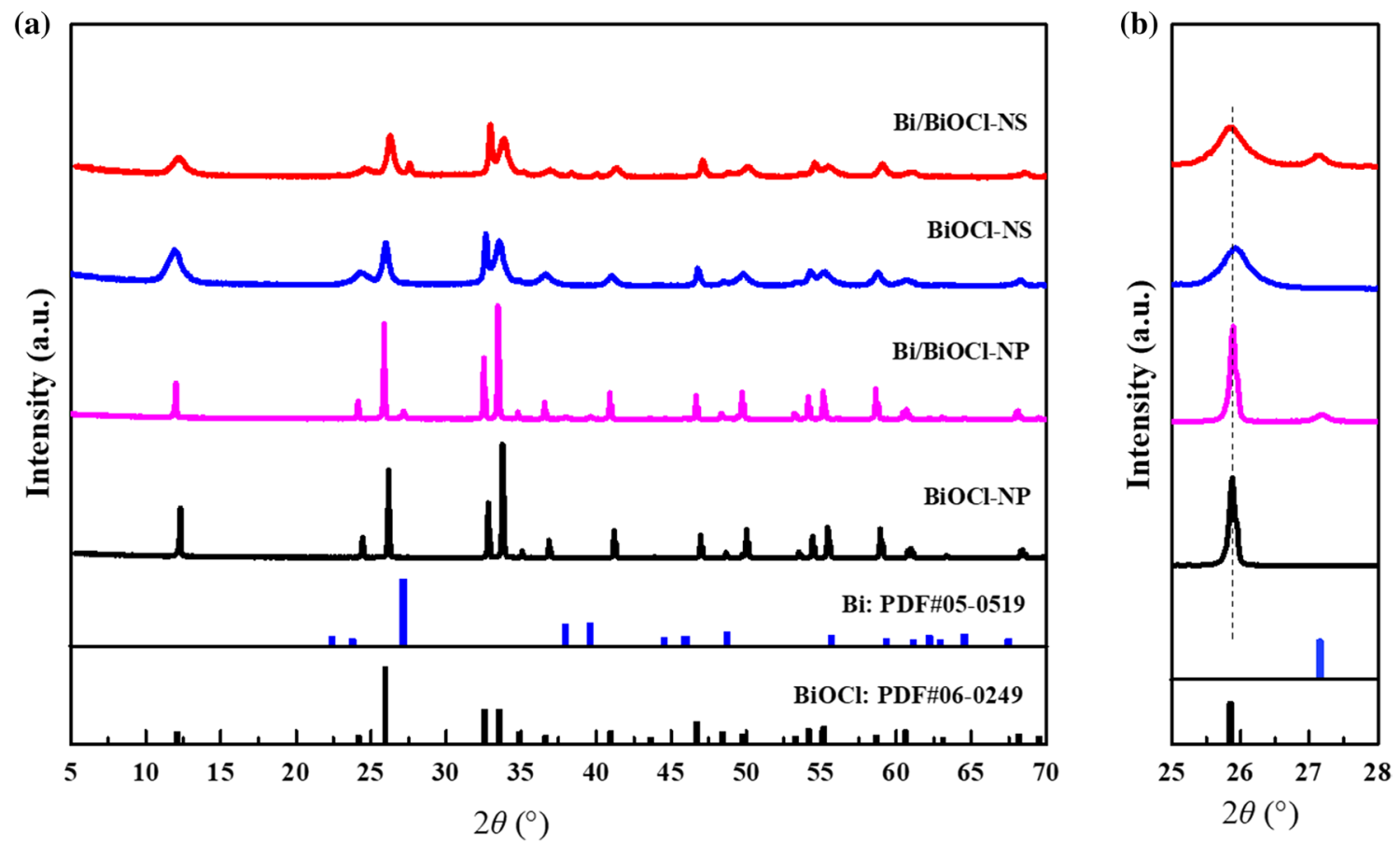

Fig. 1 a XRD spectra. b Magnification spectra from $25^{\circ}$ to $28^{\circ}$ 

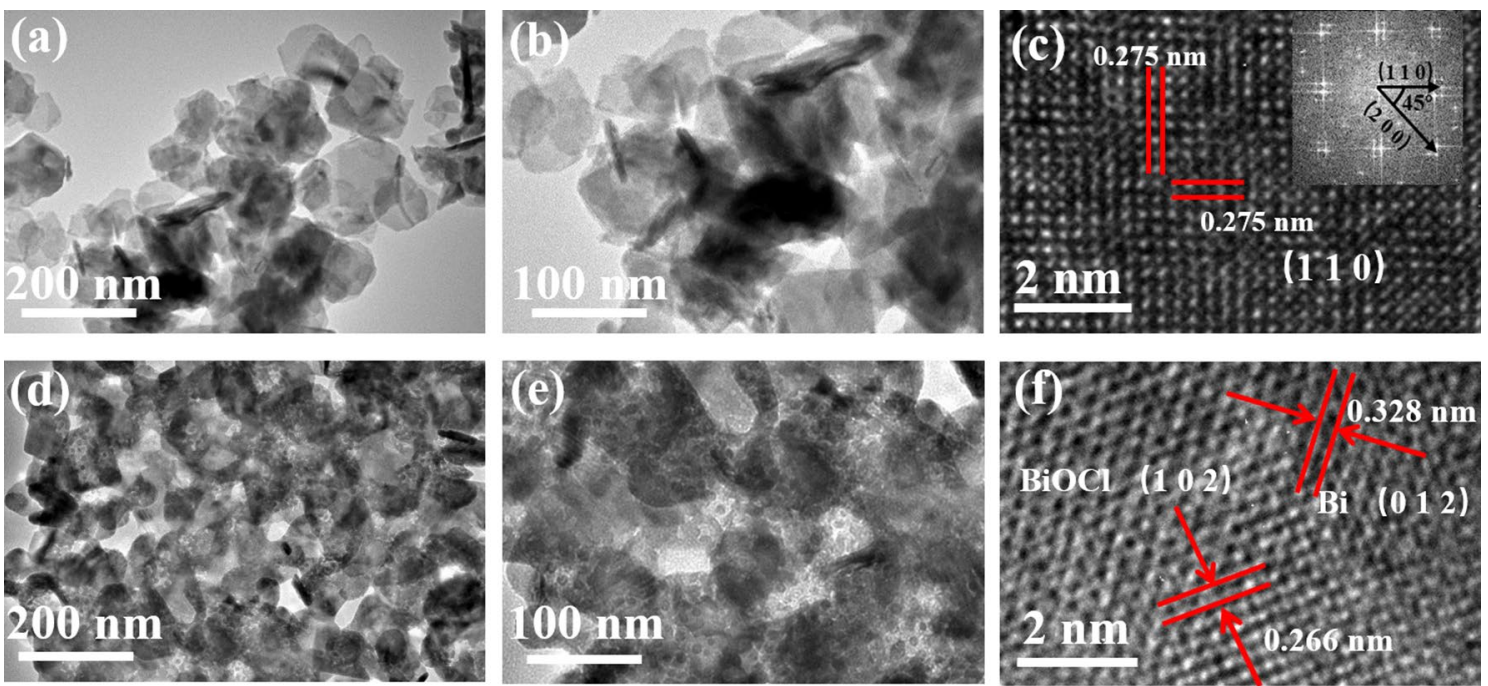

Fig. 2 TEM images of $\mathbf{a}, \mathbf{b}$ BiOCl-NS, and $\mathbf{d}, \mathbf{e}$ Bi/BiOCl-NS. HRTEM images of $\mathbf{c} \mathrm{BiOCl}-\mathrm{NS}$ and $\mathbf{f} \mathrm{Bi} / \mathrm{BiOCl}-\mathrm{NS}$

few hundred nanometers (Fig. S2). The HRTEM image of BiOCl-NS clearly shows the lattice fringes with a lattice spacing of $0.275 \mathrm{~nm}$ and an angle of $90^{\circ}$ (Fig. 2c), well matched with the (110) plane of tetragonal phase $\mathrm{BiOCl}$. This result confirmed the formation of $\mathrm{BiOCl}$ nanosheets with the exposed $\{001\}$ facets in BiOCl-NS sample. Although $\mathrm{NaBH}_{4}$ reduction destroyed part of the BiOClNS surface in the Bi/BiOCl-NS sample, it still maintained nanosheet-like morphology (Fig. 2d and Fig. S3). Besides, uniformly dispersed nanodots on the surface of $\mathrm{BiOCl}$ with less than $10 \mathrm{~nm}$ in diameter were evident (Fig. 2e). The HRTEM image of Bi/BiOCl-NS sample confirmed that these nanodots are $\mathrm{Bi}$ metal with the (012) planes of $0.328 \mathrm{~nm}$ (Fig. 2f), consistent with the result of XRD (Fig. 1a). The TEM images and XRD results of the as-prepared samples confirmed the formation of $\mathrm{BiOCl}$ nanoplates, $\mathrm{BiOCl}$ nanosheets, $\mathrm{Bi} / \mathrm{BiOCl}$ nanoplates, and $\mathrm{Bi} / \mathrm{BiOCl}$ nanosheets.

To elucidate the surface chemical properties of $\mathrm{Bi} / \mathrm{BiOCl}$ nanoplates and nanosheets, the chemical states of $\mathrm{Bi} / \mathrm{BiOCl}-$ NS and Bi/BiOCl-NP were measured by XPS (Fig. 3). The XPS survey patterns of both samples contain the peaks of $\mathrm{Bi}$, $\mathrm{O}, \mathrm{C}$, and $\mathrm{Cl}$ (Fig. 3a). Both samples were corrected by $\mathrm{C} 1 \mathrm{~s}$ at $284.8 \mathrm{eV}$. As shown in Fig. 3b, two main peaks assigned to $\mathrm{Bi} 4 f_{7 / 2}$ and $4 f_{5 / 2}$ were observed in both samples [26, 31, 32]. These two peaks can be further fitted into two pairs of $\mathrm{Bi}^{(3-x)+}(x \geq 0)$ and Bi metal. The peaks of $\mathrm{Bi} 4 f$ at $164.4 \mathrm{eV}$ and $159.1 \mathrm{eV}$ in $\mathrm{Bi} / \mathrm{BiOCl}-\mathrm{NP}$ sample were assigned to $\mathrm{Bi}$ $4 f_{7 / 2}$ and $4 f_{5 / 2}$ of $\mathrm{Bi}^{(3-x)+}(x \geq 0)$, respectively. However, these two peaks shifted to lower binding energies of $164.2 \mathrm{eV}$ and $158.9 \mathrm{eV}$ in $\mathrm{Bi} / \mathrm{BiOCl}-\mathrm{NS}$ sample, indicating a higher electron density around the $\mathrm{Bi}$ element due to the appearance of defects in $\mathrm{Bi} / \mathrm{BiOCl}-\mathrm{NS}$ [11, 33]. In addition, another two pairs of peaks at $162.6 \mathrm{eV}\left(\mathrm{Bi} 4 f_{5 / 2}\right)$ and $156.9 \mathrm{eV}\left(\mathrm{Bi} 4 f_{7 / 2}\right)$ assigned to the Bi metal showed no obvious shifts, but the relative intensities increased in $\mathrm{Bi} / \mathrm{BiOCl}-\mathrm{NS}$ sample, implying a higher concentration of $\mathrm{Bi}$ metal on the surface of $\mathrm{Bi} /$ $\mathrm{BiOCl}-\mathrm{NS}$ than that of $\mathrm{Bi} / \mathrm{BiOCl}-\mathrm{NP}$.

The $\mathrm{O} 1 s$ peaks can be fitted into two peaks in both samples (Fig. 3c). The peaks at $530.2 \mathrm{eV}$ in $\mathrm{Bi} / \mathrm{BiOCl}-\mathrm{NS}$ and $529.8 \mathrm{eV}$ in $\mathrm{Bi} / \mathrm{BiOCl}-\mathrm{NP}$ were assigned to the $\mathrm{Bi}-\mathrm{O}$ bonds $[10,32]$. O $1 s$ XPS of Bi/BiOCl-NP sample showed an obvious peak at $531.6 \mathrm{eV}$ assigned to the surface $\mathrm{OH}$ groups, which was difficult to be found in $\mathrm{Bi} / \mathrm{BiOCl}-\mathrm{NS}$ sample. The fitted peak at $532.9 \mathrm{eV}$ might be assigned to the oxygen vacancies on the surface $[10,34,35]$. Additionally, for the $\mathrm{Bi} / \mathrm{BiOCl}-\mathrm{NS}$ and $\mathrm{Bi} / \mathrm{BiOCl}-\mathrm{NP}$, there are two main peaks at $197.7 \mathrm{eV}$ and $199.5 \mathrm{eV}$ belonging to $\mathrm{Cl} 2 p$ XPS spectra (Fig. 3d) [36].

The ESR spectroscopy was used to verify the presence of oxygen vacancies. The pristine BiOCl-NS showed no signal all over the range, indicating the absence of the non-solitary pair electrons in the sample. However, $\mathrm{Bi} / \mathrm{BiOCl}-\mathrm{NS}$ and $\mathrm{Bi} /$ BiOCl-NP samples showed significant signals at $g=2.001$, which is a classic signal of oxygen vacancies (Fig. 4) [11, $12,16]$. Interestingly, the EPR signal of $\mathrm{Bi} / \mathrm{BiOCl}-\mathrm{NS}$ arising from oxygen vacancy is much stronger than that of $\mathrm{Bi} / \mathrm{BiOCl}-\mathrm{NP}$, indicating a significantly enhanced oxygen vacancy concentration of $\mathrm{Bi} / \mathrm{BiOCl}-\mathrm{NS}$. The thickness of $\mathrm{BiOCl}$ was reported to be a key factor to control the formation of surface oxygen vacancies and photocatalytic activity for $\mathrm{CO}_{2}$ reduction. This phenomenon is much obvious when $\mathrm{BiOCl}$ exposed $\{001\}$ facets due to much more oxygen atoms on $\{001\}$ facets than on other facets [37]. Specifically, the thinner nanosheets with exposed $\{001\}$ facets would induce oxygen vacancies easier on the $\mathrm{BiOCl}$ surface [38]. Therefore, it is reasonable that a thinner $\mathrm{BiOCl}$ can induce 

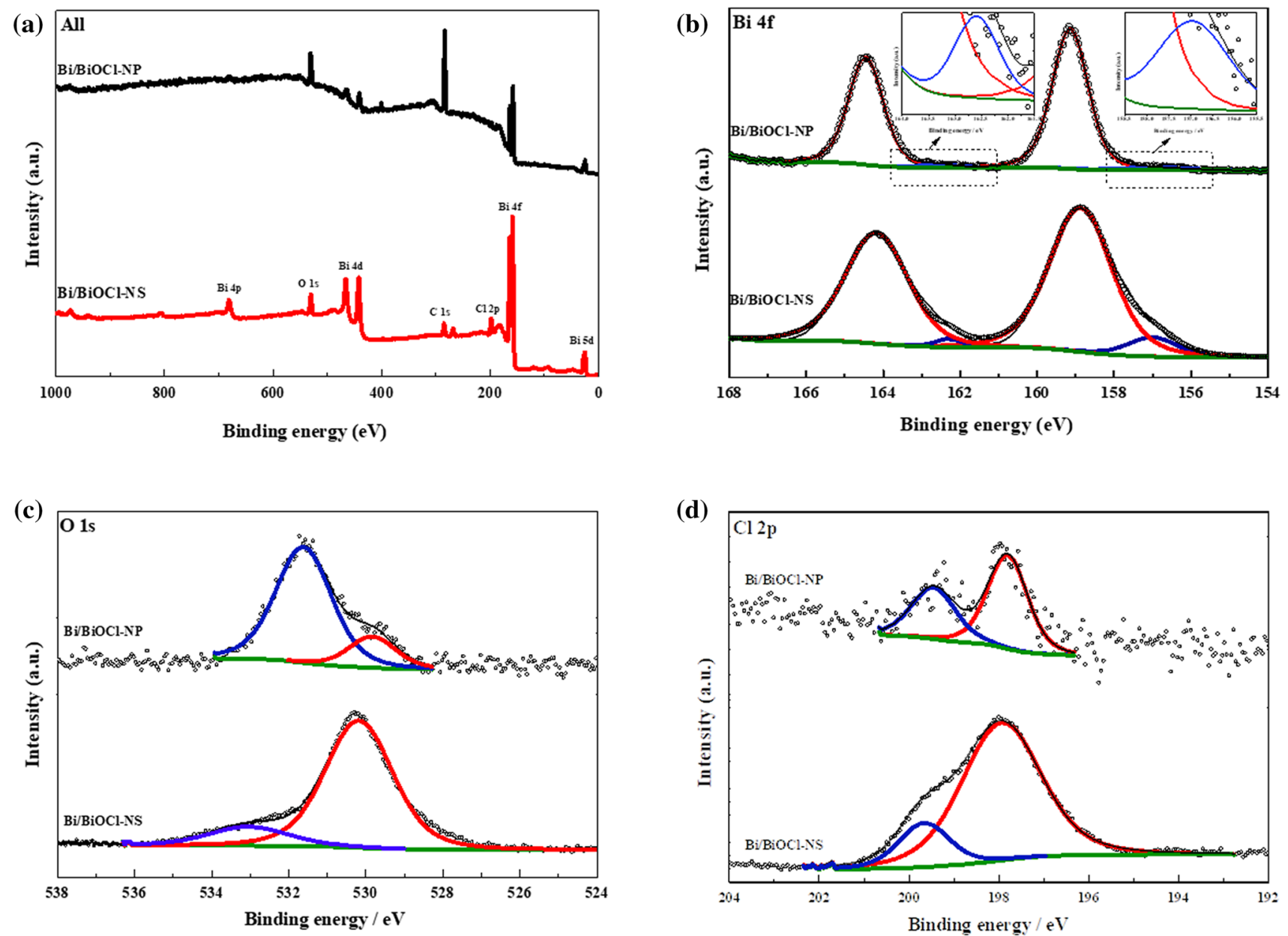

Fig. 3 a XPS survey pattern of the samples, b Bi $4 f$, $\mathbf{c} \mathrm{O} 1 s, \mathbf{d}$ Cl $2 p$ XPS of Bi/BiOCl-NP and Bi/BiOCl-NS

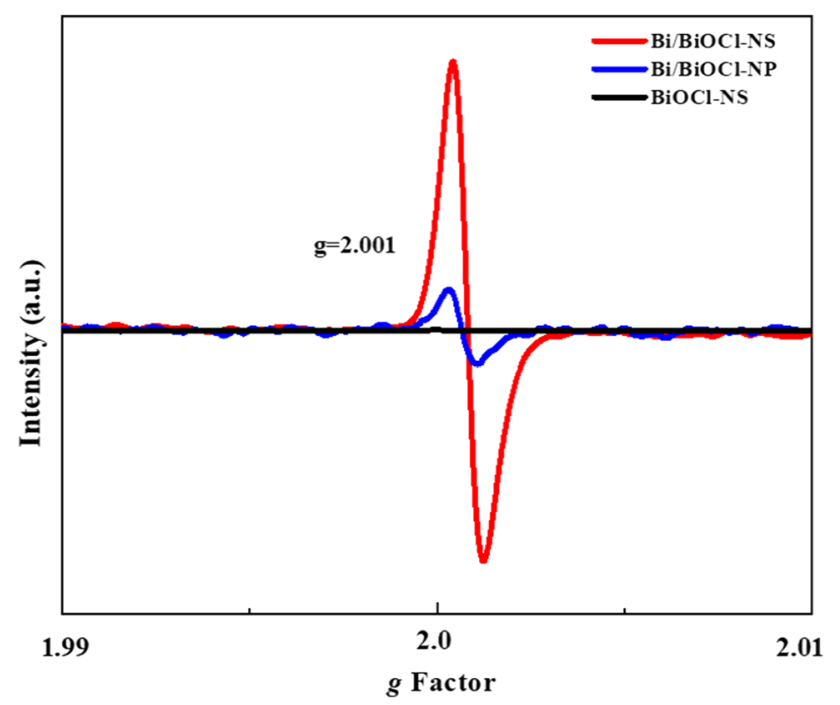

Fig. 4 EPR spectra of BiOCl-NS, Bi/BiOCl-NS, and Bi/BiOCl-NP at room temperature higher amount of oxygen vacancies after $\mathrm{NaBH}_{4}$ reduction to form $\mathrm{Bi} / \mathrm{BiOCl}$ enriched oxygen vacancies. Generally, a high amount of oxygen vacancies on the surface of catalysts is believed to show better activity due to their involvement in new photoexcitation processes [11, 13, 39].

The optical absorption properties of the as-prepared samples were measured using UV-Vis diffuse reflectance spectra. It can be seen in Fig. 5 that the pristine $\mathrm{BiOCl}$ shows absorption edge at around $360 \mathrm{~nm}$, indicating a band gap of $3.4 \mathrm{eV}$, which is consistent with other reports [25, 26]. $\mathrm{Bi} / \mathrm{BiOCl}-\mathrm{NS}$ sample showed similar absorption edge the pristine $\mathrm{BiOCl}$. However, an absorption tail extended to the whole visible light range. This is attributed to the formation of oxygen vacancies, which is consistent with the results of XPS and EPR (Figs. 3c, 4) [33]. In Bi/BiOCl-NP, the absorption peak centered at $500 \mathrm{~nm}$ instead of the absorption tail was found due to the formation of Bi particles with its surface plasmon resonance (SPR) effect. Based on the analysis above, Bi/BiOCl-NS enriched with oxygen vacancies was formed during the reducing process. 


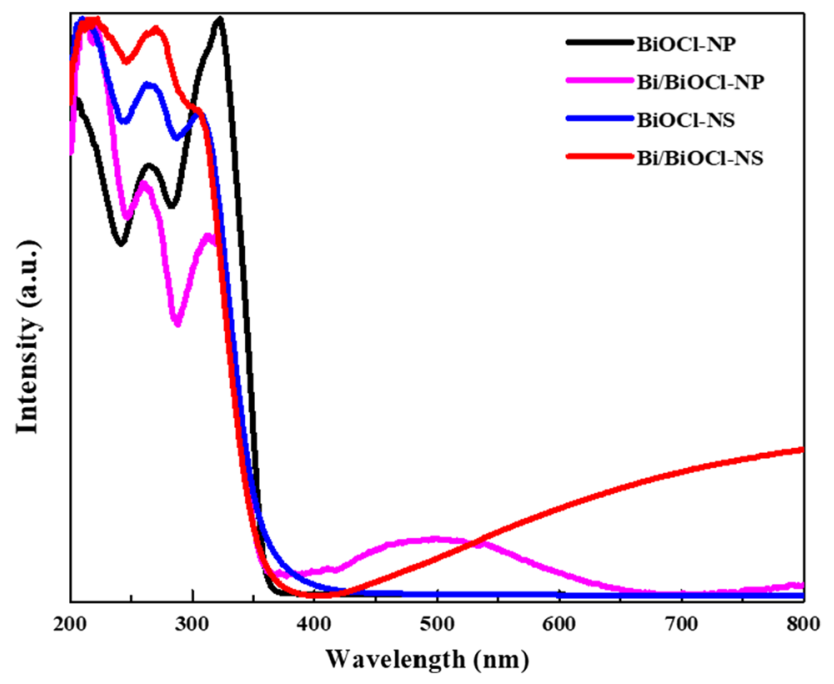

Fig. 5 UV-Vis absorbance spectra of BiOCl-NP, BiOCl-NS, Bi/ BiOCl-NP, and BiOCl-NS

The photocatalytic activities of $\mathrm{CO}_{2}$ reduction over the as-prepared samples were carried out in a close system using 300-W Xe lamp as a light source with $\mathrm{H}_{2} \mathrm{O}$ vapor. The detected products were $\mathrm{H}_{2}, \mathrm{CO}$, and $\mathrm{CH}_{4}$. As shown in Fig. 6, the pristine BiOCl-NP showed the lowest photocatalytic activities of all the detectable products. However, pristine BiOCl-NS sample showed enhanced photocatalytic activity, which is consistent with Ref. [12]. It is noted that $\mathrm{Bi} / \mathrm{BiOCl}-\mathrm{NS}$ sample showed the highest photocatalytic activity of $\mathrm{CO}_{2}$ reduction. The formation amounts of $\mathrm{CO}, \mathrm{CH}_{4}$ and $\mathrm{H}_{2}$ in $8 \mathrm{~h}$ are $38 \mu \mathrm{mol} / \mathrm{g}, 3.6 \mu \mathrm{mol} / \mathrm{g}$, and $1.9 \mu \mathrm{mol} / \mathrm{g}$, respectively, which are $2.7,5.1$, and 1.4 times of pristine $\mathrm{BiOCl}-\mathrm{NS}$ sample. Bi/BiOCl-NS with thinner $\mathrm{BiOCl}$ nanosheets showed much higher activity in all products than $\mathrm{Bi} / \mathrm{BiOCl}-\mathrm{NP}$ with thicker $\mathrm{BiOCl}$ nanoplates. The

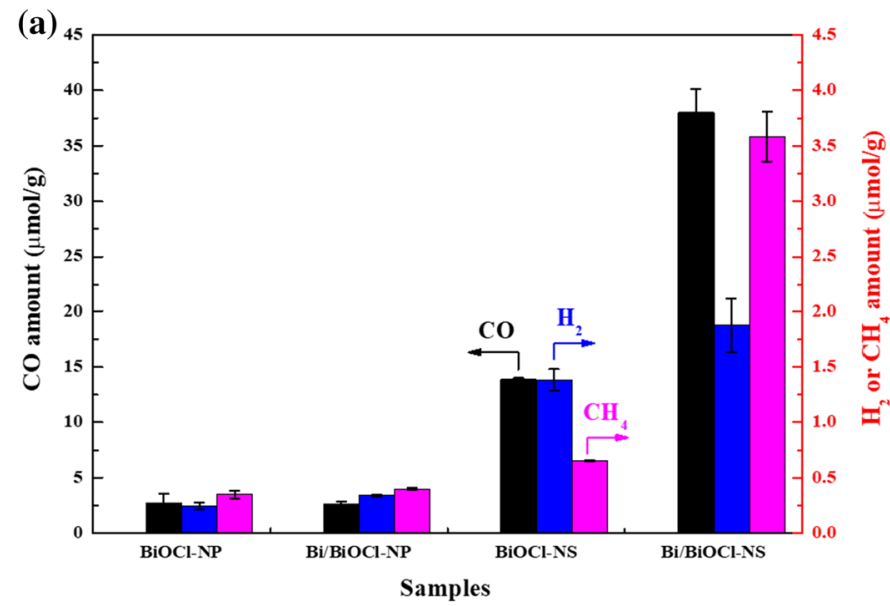

results also showed that $\mathrm{Bi}$ metal on $\mathrm{BiOCl}$ showed an overall enhancement of activity compared to bare $\mathrm{BiOCl}$ in both nanoplates and nanosheets. Higher oxygen vacancies in $\mathrm{Bi} /$ BiOCl-NS showed higher formation rates of $\mathrm{CH}_{4}$ and $\mathrm{CO}$, while no obvious enhancement of $\mathrm{H}_{2}$. It should be noted that the enhancement of photocatalytic activity was not due to the SPR effect because the sample of Bi/BiOCl-NP with Bi SPR peak showed no activity under visible light irradiation $(\lambda>420 \mathrm{~nm})$.

Compared with some typical photocatalysts that were reported under similar reaction conditions, photocatalysts synthesized in this study showed moderate activity for formations of $\mathrm{CO}$ and $\mathrm{CH}_{4}$ and good activity for suppressing $\mathrm{H}_{2}$ formation (Table $\mathrm{S} 1$ ). The time for each product in Fig. $6 \mathrm{~b}$ showed steadily increasing formation of these products during photoirradiation. The photocatalytic activities of $\mathrm{Bi} / \mathrm{BiOCl}$ during the first two cycles showed a significant decrease, which might be due to the further reduction of $\mathrm{BiOCl}$ and/or disappearance of oxygen vacancies. However, stable photocatalytic activities were observed during the third cycle (Fig. S4).

The selectivity of $\left(\mathrm{CO}+\mathrm{CH}_{4}\right)$ was higher than $90 \%$, whereas $\mathrm{H}_{2}$ selectivity was less than $10 \%$ in all cases, indicating that water splitting during the reaction was heavily suppressed. $\mathrm{CH}_{4}$ selectivity showed enhancement after $\mathrm{Bi}$ metal and oxygen vacancies formed on both BiOCl-NP and BiOCl-NS (Fig. S5), which indicated that Bi metal and oxygen vacancies might be beneficial for the formation of $\mathrm{CH}_{4}$. $\mathrm{CH}_{4}$ selectivity was enhanced by $3 \%$ over $\mathrm{Bi} / \mathrm{BiOCl}-\mathrm{NP}$ as compared to that over bare BiOCl-NP. This enhancement was increased to $\sim 12 \%$ between $\mathrm{BiOCl}-\mathrm{NS}$ and $\mathrm{Bi} / \mathrm{BiOCl}-$ NS. Considering that the concentration of oxygen vacancies was much higher in $\mathrm{Bi} / \mathrm{BiOCl}-\mathrm{NS}$, oxygen vacancy might be the important factor for $\mathrm{CH}_{4}$ selectivity during photocatalytic $\mathrm{CO}_{2}$ reduction.

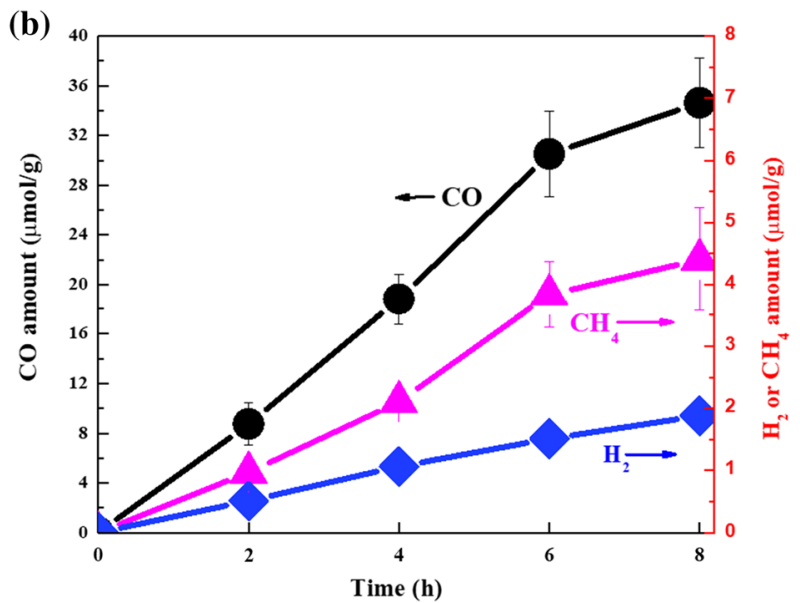

Fig. 6 a Generations of $\mathrm{CO}, \mathrm{CH}_{4}$, and $\mathrm{H}_{2}$ over $\mathrm{BiOCl}-\mathrm{NP}$, Bi/BiOCl-NP, BiOCl-NS, and Bi/BiOCl-NS under UV-Vis irradiation for 8 h. b Time-dependent photocatalytic evolution of products over $\mathrm{Bi} / \mathrm{BiOCl}-\mathrm{NS}$ 
Five blank tests and an isotopic experiment were performed to confirm the photocatalytic process. The results revealed that $\mathrm{BiOCl}$ with $\mathrm{Bi}$ as cocatalyst in $\mathrm{CO}_{2}$ and $\mathrm{H}_{2} \mathrm{O}$ atmosphere under photoirradiation can consistently achieve the significant formation of $\mathrm{CO}$, which indicated that the reaction process should be a photocatalytic process (Fig. 7a). The isotopic experiments with ${ }^{13} \mathrm{CO}_{2}$ and $\mathrm{H}_{2} \mathrm{O}$ showed that the ${ }^{13} \mathrm{CO}$ and ${ }^{13} \mathrm{CH}_{4}$ were formed from ${ }^{13} \mathrm{CO}_{2}$ during the photoirradiation (Fig. 7b). This further confirmed that $\mathrm{CO}_{2}$ was photocatalytically reduced to carbon sources during the reaction.

The specific surface area should show significant effect when comparing the activity between BiOCl-NP and BiOClNS. However, when the $\mathrm{NaBH}_{4}$ was used to reduce both BiOCl-NP and BiOCl-NS, the enhancement of activities between $\mathrm{Bi} / \mathrm{BiOCl}-\mathrm{NP}$ and $\mathrm{Bi} / \mathrm{BiOCl}-\mathrm{NS}$ was obviously different. Bi/BiOCl-NS showed much higher enhancement than $\mathrm{Bi} / \mathrm{BiOCl}-\mathrm{NP}$ with same reduction concentration of $\mathrm{NaBH}_{4}$. Specifically, $\mathrm{CO}$ formation over $\mathrm{Bi} / \mathrm{BiOCl}-\mathrm{NS}$ was 2.7 times higher than that over BiOCl-NS. However, no obvious enhancement was found over BiOCl-NP and Bi/BiOCl-NP (Fig. 6a). Besides, $\mathrm{CH}_{4}$ formation over $\mathrm{Bi} / \mathrm{BiOCl}-\mathrm{NS}$ was 5.5 times higher than that over BiOCl-NS. However, the enhancement was only 1.1 over $\mathrm{BiOCl}-\mathrm{NP}$ and $\mathrm{Bi} / \mathrm{BiOCl}-$ NP. Therefore, specific surface area should not be the main factor for the enhancement of activity.

PL spectra were acquired to study the generation, transfer, and recombination of electron-hole pairs. Figure 8 shows the PL spectra of as-prepared samples with excitation at $250 \mathrm{~nm}$. BiOCl-NP sample showed the highest PL intensity, indicating highest photogenerated electron-hole pairs recombination rate. Therefore, this sample showed the lowest photocatalytic activity for $\mathrm{CO}_{2}$ reduction. Significant decrease of PL intensities of Bi/BiOCl-NP and Bi/BiOCl-NS

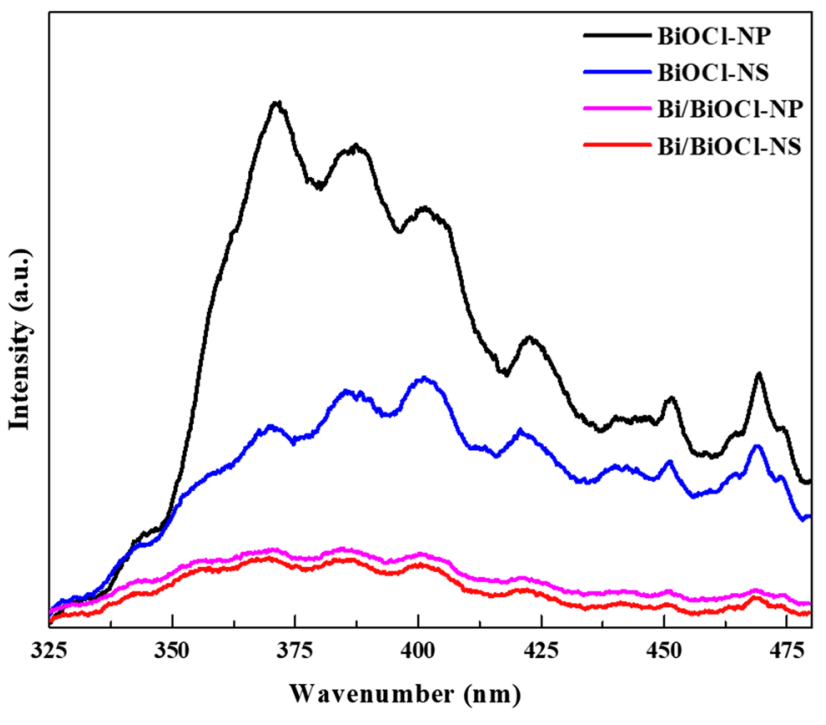

Fig. 8 PL spectra at room temperature

samples implied that the in situ reduced Bi showed a significant increase in the separation rate of electron-hole pairs. It was reported in our previous works that metal $\mathrm{Bi}$ is a good medium for conducting electrons [40, 41]. When metal Bi is loaded on the surface of the $\mathrm{BiOCl}$, it can effectively improve the transfer efficiency of electrons. In turn, more electrons are involved in the reduction reaction. Interestingly, BiOCl-NS showed lower PL intensity than BiOCl-NP. This means that BiOCl-NS enriched with oxygen vacancies showed a better efficiency for separation of electron-hole pairs.

Photocurrent tests were performed to further study the recombination rate of photogenerated charge carriers (Fig. 9). All samples showed reproducible on-off effects
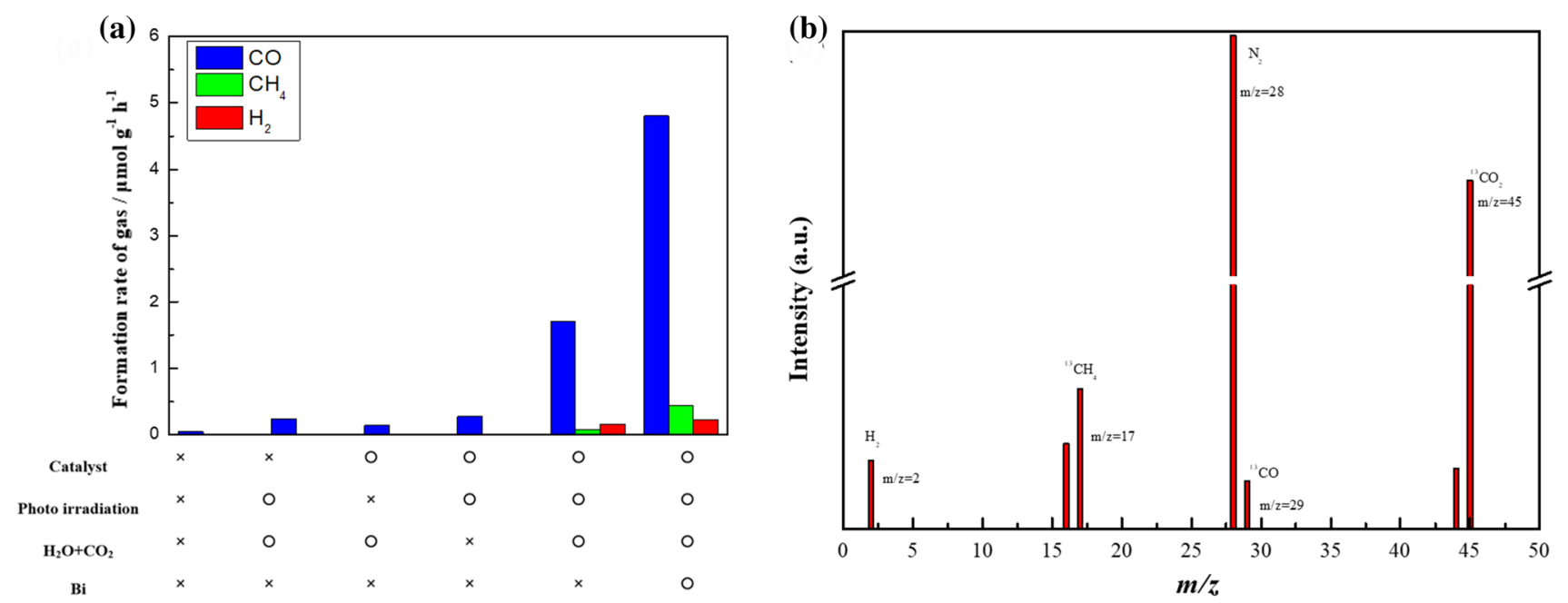

Fig. 7 a Blank tests over $\mathrm{Bi} / \mathrm{BiOCl}-\mathrm{NS}$. b MS of the $\mathrm{CO}_{2}$ reduction reactions using ${ }^{13} \mathrm{CO}_{2}$ 


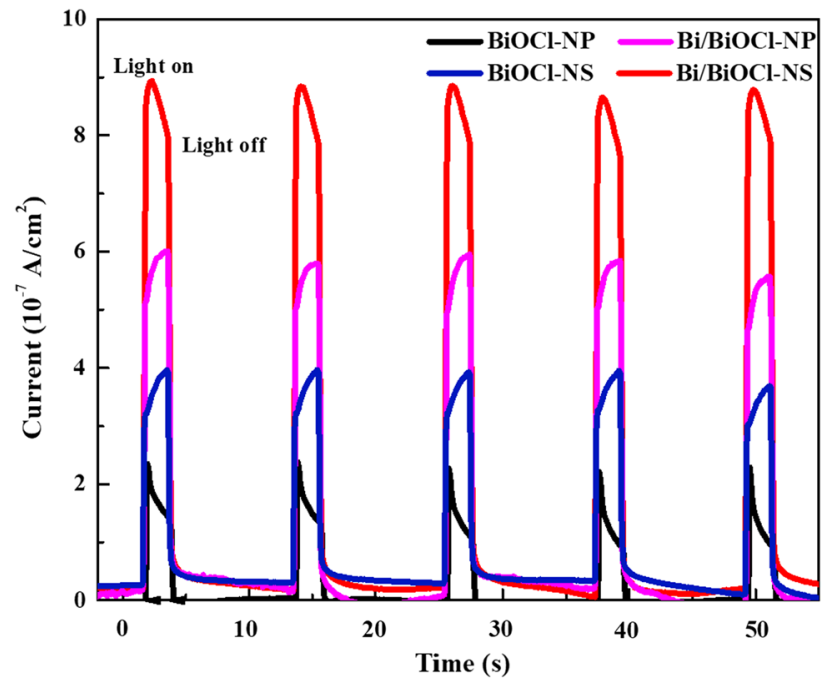

Fig. 9 Transient photocurrent responses of BiOCl-NP, BiOCl-NS, Bi/ $\mathrm{BiOCl}-\mathrm{NP}$, and $\mathrm{Bi} / \mathrm{BiOCl}-\mathrm{NS}$

with the photoirradiation turned on and off. BiOCl-NP sample showed the lowest photocurrent density, whereas BiOCl-NS showed the enhanced photocurrent density. This indicated that thinner $\mathrm{BiOCl}$ could be beneficial for the photogenerated charge mobility, consistent with the photocatalytic activity for $\mathrm{CO}_{2}$ reduction. It is found that $\mathrm{Bi}$ loading on $\mathrm{BiOCl}$ showed obvious enhancement of photocurrent density. Among all tested samples, the $\mathrm{Bi} / \mathrm{BiOCl}-\mathrm{NS}$ showed the highest photocurrent density of $0.87 \mu \mathrm{A} / \mathrm{cm}^{2}$, which was about 2 times than that of pristine BiOCl-NS $\left(0.37 \mu \mathrm{A} / \mathrm{cm}^{2}\right)$ and $47 \%$ higher than that of Bi/BiOCl-NP $\left(0.59 \mu \mathrm{A} / \mathrm{cm}^{2}\right)$. Note that the trend of photocurrent density is consistent with that of the photocatalytic activity of $\mathrm{CO}_{2}$ reduction, implying that the separation of charge carriers plays an important role during photocatalysis.

Based on the above characterization and discussion, $\mathrm{Bi}$ reduction from $\mathrm{BiOCl}$ nanosheets with enriched oxygen vacancies is believed to play a crucial role in photocatalytic $\mathrm{CO}_{2}$ reduction. $\mathrm{NaBH}_{4}$ not only acted as a reductant to form $\mathrm{Bi}$ on $\mathrm{BiOCl}$, but also induced the formation of oxygen vacancies. Therefore, the photocatalytic activity for $\mathrm{CO}_{2}$ reduction was dramatically enhanced compared to the bare $\mathrm{BiOCl}$ nanosheets. $\mathrm{Bi}$ acts as a metal to effectively enhance the separation of electrons and holes. Such effect could be further enhanced with oxygen vacancies, and therefore, $\mathrm{Bi} / \mathrm{BiOCl}$ nanosheets showed much higher photocatalytic activity for $\mathrm{CO}_{2}$ reduction than $\mathrm{Bi} / \mathrm{BiOCl}$ nanoplates (Scheme 1).

\section{Conclusions}

In summary, we fabricated $\mathrm{Bi} / \mathrm{BiOCl}$ nanosheets enriched with oxygen vacancies to show enhanced photocatalytic activity for $\mathrm{CO}_{2}$ reduction. Pure $\mathrm{BiOCl}$ nanoplates with higher thickness showed the lowest photocatalytic activity, and the enhancement of photocatalytic activity was limited even with the formation of $\mathrm{Bi}$ as cocatalysts on $\mathrm{BiOCl}$ nanoplates using $\mathrm{NaBH}_{4}$ as a reductant. On the other hand, such effect of enhancement of photocatalytic activity was dramatically enlarged when Bi metal was formed on lower thickness of $\mathrm{BiOCl}$ nanosheets. It is also believed that the enriched oxygen vacancies are a key factor for the further enhancement of photocatalytic activity for $\mathrm{CO}_{2}$ reduction. This work suggested that the Bi particles enriched with oxygen vacancies on $\mathrm{BiOCl}$ nanosheets play crucial roles in converting solar energy into chemical energy.

Acknowledgements This research was financially supported by the Sichuan Provincial International Cooperation Project (No. 2019YFH0164), Chengdu International Cooperation Project (No. 2019-GH02-00056-HZ), and the National Natural Science Foundation of China (No. U1862111). Ying Zhou thanks the Cheung Kong

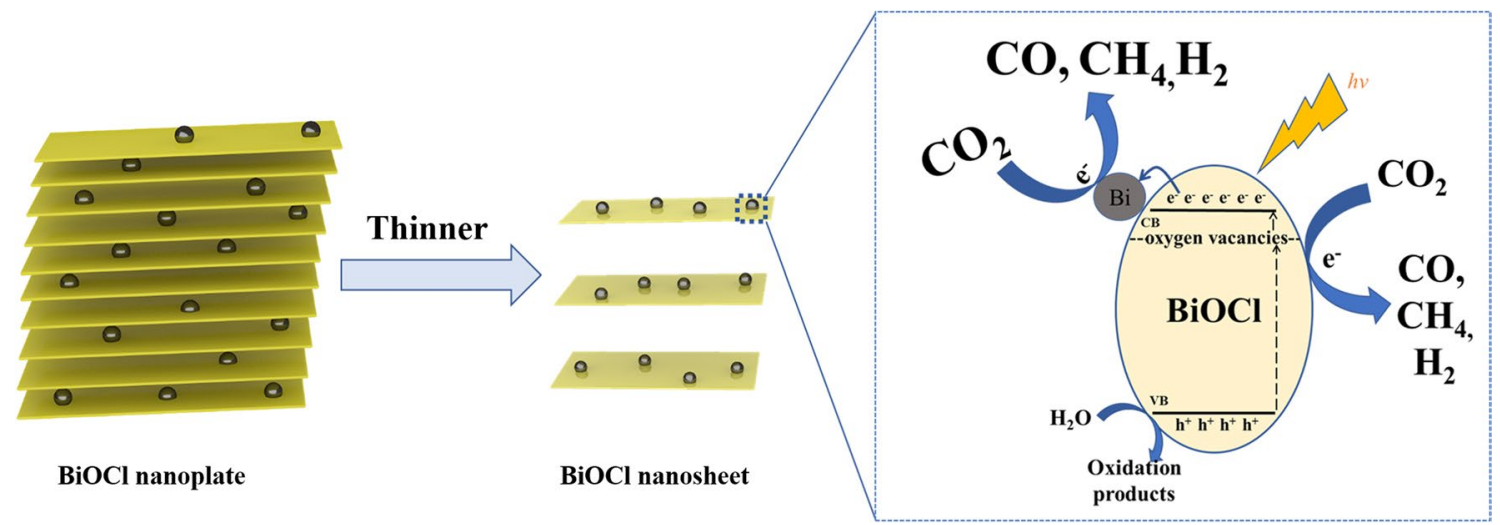

Scheme 1 Schematic illustration of the proposed mechanism of photocatalytic $\mathrm{CO}_{2}$ reduction over $\mathrm{Bi} / \mathrm{BiOCl}$ 
Scholars Program of China and JSPS Invitational Fellowships for Research in Japan.

Open Access This article is licensed under a Creative Commons Attribution 4.0 International License, which permits use, sharing, adaptation, distribution and reproduction in any medium or format, as long as you give appropriate credit to the original author(s) and the source, provide a link to the Creative Commons licence, and indicate if changes were made. The images or other third party material in this article are included in the article's Creative Commons licence, unless indicated otherwise in a credit line to the material. If material is not included in the article's Creative Commons licence and your intended use is not permitted by statutory regulation or exceeds the permitted use, you will need to obtain permission directly from the copyright holder. To view a copy of this licence, visit http://creativecommons.org/licenses/by/4.0/.

\section{References}

1. Zvereva EL, Kozlov MV (2006) Consequences of simultaneous elevation of carbon dioxide and temperature for plant-herbivore interactions: a metaanalysis. Glob Change Biol 12(1):27-41

2. Khasnis AA, Nettleman MD (2005) Global warming and infectious disease. Arch Med Res 36(6):689-696

3. Kawai T, Sakata T (1980) Conversion of carbohydrate into hydrogen fuel by a photocatalytic process. Nature 286(5772):474-476

4. Nie N, Zhang LY, Fu JW et al (2018) Self-assembled hierarchical direct $\mathrm{Z}$-scheme $\mathrm{g}-\mathrm{C}_{3} \mathrm{~N}_{4} / \mathrm{ZnO}$ microspheres with enhanced photocatalytic $\mathrm{CO}_{2}$ reduction performance. Appl Surf Sci 441:12-22

5. Low J, Cheng B, Yu JG (2017) Surface modification and enhanced photocatalytic $\mathrm{CO}_{2}$ reduction performance of $\mathrm{TiO}_{2}$ : a review. Appl Surf Sci 392:658-686

6. Dong CY, Hu SC, Xing MY et al (2018) Enhanced photocatalytic $\mathrm{CO}_{2}$ reduction to $\mathrm{CH}_{4}$ over separated dual co-catalysts: $\mathrm{Au}$ and $\mathrm{RuO}_{2}$. Nanotechnology 29(15):154005

7. Dong CY, Lian C, Hu SC et al (2018) Size-dependent activity and selectivity of carbon dioxide photocatalytic reduction over platinum nanoparticles. Nat Commun 9:1252

8. Xing MY, Zhou Y, Dong CY et al (2018) Modulation of the reduction potential of $\mathrm{TiO}_{2-x}$ by fluorination for efficient and selective $\mathrm{CH}_{4}$ generation from $\mathrm{CO}_{2}$ photoreduction. Nano Lett 18(6):3384-3390

9. Liu HM, Meng XG, Dao TD et al (2017) Light assisted $\mathrm{CO}_{2}$ reduction with methane over $\mathrm{SiO}_{2}$ encapsulated $\mathrm{Ni}$ nanocatalysts for boosted activity and stability. J Mater Chem A 5(21):10567-10573

10. Zhang L, Wang WZ, Jiang D et al (2015) Photoreduction of $\mathrm{CO}_{2}$ on $\mathrm{BiOCl}$ nanoplates with the assistance of photoinduced oxygen vacancies. Nano Res 8(3):821-831

11. Ma ZY, Li PH, Ye LQ et al (2017) Oxygen vacancies induced exciton dissociation of flexible $\mathrm{BiOCl}$ nanosheets for effective photocatalytic $\mathrm{CO}_{2}$ conversion. J Mater Chem A 5(47):24995-25004

12. Jin JR, Wang YJ, He T (2015) Preparation of thickness-tunable $\mathrm{BiOCl}$ nanosheets with high photocatalytic activity for photoreduction of $\mathrm{CO}_{2}$. RSC Adv 5(121):100244-100250

13. Guan ML, Xiao C, Zhang J et al (2013) Vacancy associates promoting solar-driven photocatalytic activity of ultrathin bismuth oxychloride nanosheets. J Am Chem Soc 135(28):10411-10417

14. Li BX, Shao LZ, Zhang BS et al (2017) Understanding sizedependent properties of $\mathrm{BiOCl}$ nanosheets and exploring more catalysis. J Colloid Interface Sci 505:653-663

15. Cai YJ, Li DY, Sun JY et al (2018) Synthesis of BiOCl nanosheets with oxygen vacancies for the improved photocatalytic properties. Appl Surf Sci 439:697-704
16. $\mathrm{Li} \mathrm{H}, \mathrm{Li}$ J, Ai ZH et al (2018) Oxygen vacancy-mediated photocatalysis of $\mathrm{BiOCl}$ : reactivity, selectivity, and perspectives. Angew Chem Int Ed 57(1):122-138

17. Li BX, Shao LZ, Wang RS et al (2018) Interfacial synergism of $\mathrm{Pd}$-decorated $\mathrm{BiOCl}$ ultrathin nanosheets for the selective oxidation of aromatic alcohols. J Mater Chem A 6(15):6344-6355

18. Bai S, Li XY, Kong Q et al (2015) Toward enhanced photocatalytic oxygen evolution: synergetic utilization of plasmonic effect and Schottky junction via interfacing facet selection. Adv Mater 27(22):3444-3452

19. Di J, Xia JX, Yin S et al (2014) One-pot solvothermal synthesis of $\mathrm{Cu}$-modified $\mathrm{BiOCl}$ via a $\mathrm{Cu}$-containing ionic liquid and its visible-light photocatalytic properties. RSC Adv 4(27):14281-14290

20. Cao X, Chen Z, Lin R et al (2018) A photochromic composite with enhanced carrier separation for the photocatalytic activation of benzylic C-H bonds in toluene. Nat Catal 1(9):704-710

21. de la Garza-Galván M, Zambrano-Robledo P, Vazquez-Arenas $\mathrm{J}$ et al (2019) In situ synthesis of Au-decorated $\mathrm{BiOCl} / \mathrm{BiVO}_{4}$ hybrid ternary system with enhanced visible-light photocatalytic behavior. Appl Surf Sci 487:743-754

22. Cui ZK, Song HT, Ge SX et al (2019) Fabrication of BiOCl/ $\mathrm{BiOBr}$ hybrid nanosheets with enhanced superoxide radical dominating visible light driven photocatalytic activity. Appl Surf Sci 467-468:505-513

23. Lu Y, Song JM, Li WF et al (2020) Preparation of $\mathrm{BiOCl} / \mathrm{Bi}_{2} \mathrm{~S}_{3}$ composites by simple ion exchange method for highly efficient photocatalytic reduction of $\mathrm{Cr}^{6+}$. Appl Surf Sci 506:145000

24. Wang H, Zhang WD, Li XW et al (2018) Highly enhanced visible light photocatalysis and in situ FT-IR studies on Bi metal@ defective $\mathrm{BiOCl}$ hierarchical microspheres. Appl Catal B Environ 225:218-227

25. Weng SX, Chen BB, Xie LY et al (2013) Facile in situ synthesis of a $\mathrm{Bi} / \mathrm{BiOCl}$ nanocomposite with high photocatalytic activity. J Mater Chem A 1(9):3068-3075

26. Yu Cao CY, Liu H et al (2014) A Bi/BiOCl heterojunction photocatalyst with enhanced electron-hole separation and excellent visible light photodegrading activity. J Mater Chem A 2(6):1677-1681

27. Hu JJ, Xu GQ, Wang JW et al (2014) Photocatalytic properties of $\mathrm{Bi} / \mathrm{BiOCl}$ heterojunctions synthesized using an in situ reduction method. New J Chem 38(10):4913-4921

28. Hu JJ, Xu GQ, Wang JW et al (2015) Photocatalytic property of a $\mathrm{Bi}_{2} \mathrm{O}_{3}$ nanoparticle modified $\mathrm{BiOCl}$ composite with a nanolayered hierarchical structure synthesized by in situ reactions. Dalton Trans 44(12):5386-5395

29. Li Q, Sun ZX, Wang HQ et al (2018) Insight into the enhanced $\mathrm{CO}_{2}$ photocatalytic reduction performance over hollow-structured Bi-decorated g- $\mathrm{C}_{3} \mathrm{~N}_{4}$ nanohybrid under visible-light irradiation. $\mathrm{J}$ CO2 Util 28:126-136

30. Ye LQ, Deng Y, Wang L et al (2019) Bismuth-based photocatalysts for solar photocatalytic carbon dioxide conversion. Chemsuschem 12(16):3671-3701

31. Yu SX, Zhang YH, Li M et al (2017) Non-noble metal Bi deposition by utilizing $\mathrm{Bi}_{2} \mathrm{WO}_{6}$ as the self-sacrificing template for enhancing visible light photocatalytic activity. Appl Surf Sci 391:491-498

32. Sun YJ, Zhao ZW, Dong F et al (2015) Mechanism of visible light photocatalytic $\mathrm{NO}_{x}$ oxidation with plasmonic Bi cocatalystenhanced $(\mathrm{BiO})_{2} \mathrm{CO}_{3}$ hierarchical microspheres. Phys Chem Chem Phys 17(16):10383-10390

33. Li M, Zhang YH, Li XW et al (2018) Nature-derived approach to oxygen and chlorine dual-vacancies for efficient photocatalysis and photoelectrochemistry. ACS Sustain Chem Eng 6(2):2395-2406

34. Ding LY, Wei RJ, Chen H et al (2015) Controllable synthesis of highly active $\mathrm{BiOCl}$ hierarchical microsphere self-assembled 
by nanosheets with tunable thickness. Appl Catal B Environ 172-173:91-99

35. Armelao L, Bottaro G, MacCato C et al (2012) Bismuth oxychloride nanoflakes: interplay between composition-structure and optical properties. Dalton Trans 41(18):5480

36. Zhao ZY, Cao YH, Dong F et al (2019) The activation of oxygen through oxygen vacancies in $\mathrm{BiOCl} / \mathrm{PPy}$ to inhibit toxic intermediates and enhance the activity of photocatalytic nitric oxide removal. Nanoscale 11(13):6360-6367

37. Li LS, Sun NJ, Huang YY et al (2008) Topotactic transformation of single-crystalline precursor discs into disc-like $\mathrm{Bi}_{2} \mathrm{~S}_{3}$ nanorod networks. Adv Funct Mater 18(8):1194-1201

38. Ye LQ, Zan L, Tian LH et al (2011) The 001 facets-dependent high photoactivity of $\mathrm{BiOCl}$ nanosheets. Chem Commun 47(24):6951-6953

39. Li H, Shi JG, Zhao K et al (2014) Sustainable molecular oxygen activation with oxygen vacancies on the 001 facets of $\mathrm{BiOCl}$ nanosheets under solar light. Nanoscale 6(23):14168-14173

40. Zhang XJ, Yu S, Liu Y et al (2017) Photoreduction of non-noble metal $\mathrm{Bi}$ on the surface of $\mathrm{Bi}_{2} \mathrm{WO}_{6}$ for enhanced visible light photocatalysis. Appl Surf Sci 396:652-658

41. Zhou Y, Li W, Zhang Q et al (2017) Non-noble metal plasmonic photocatalysis in semimetal bismuth films for photocatalytic NO oxidation. Phys Chem Chem Phys 19(37):25610-25616

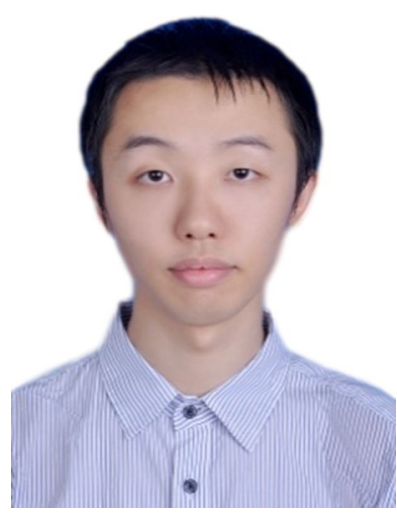

Zeai Huang is a lecturer of Southwest Petroleum University. After graduating from Kyoto University with a Ph.D. in Japan in 2018 , he conducted postdoctoral research in National Institute of Advanced Industrial Science and Technology (AIST) in Japan. In 2018, he joined Southwest Petroleum University. He is mainly engaged in research of highvalue utilization of unconventional natural gas. He has pub- lished more than 20 SCI papers, cited more than 700, with an $\mathrm{H}$ index of 13 .

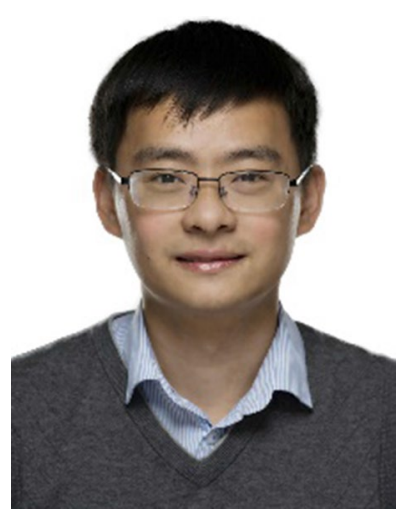

Ying Zhou is a professor in Southwest Petroleum University. Involved in the National Hundred and Thousand Talents Project, young scholars of the "Yangtze River Scholars Award Program", Humboldt scholars of Germany, scholars invited by JSPS of Japan, chair professor of Kyoto University. After graduating from the University of Zurich (UZH) with a Ph.D. in Switzerland in 2010, he conducted postdoctoral research under the funding of the Outstanding Youth Fund of the University of Zurich and then engaged in research at the Karlsruhe Institute of Technology (KIT) in Germany. His research team is mainly engaged in researches including resource utilization of hydrogen energy and hydrogen sulfide, high-value utilization of unconventional natural gas, oil-water separation materials, industrial waste gas purification and resource utilization, etc. He has published more than 120 SCI papers, cited more than 3400, with an $\mathrm{H}$ index of 34. 